\title{
On Interface Rate Allocation for a Fiber Aided Wireless Network Architecture
}

\author{
Siddharth Ray, Muriel Médard and Lizhong Zheng \\ Laboratory for Information and Decision Systems \\ Massachusetts Institute of Technology, Cambridge, MA 02139. \\ Email: sray@mit.edu, medard@mit.edu, lizhong@mit.edu
}

\begin{abstract}
The concept of a fiber aided wireless network architecture (FAWNA) is introduced in [Ray et al., Allerton 2005], which allows high-speed mobile connectivity by leveraging the speed of optical networks. Reference [Ray et al., ISIT 2006] considers a single-input, multiple-output (SIMO) FAWNA: A SIMO wireless channel interfaced with an optical fiber through wireless-optical interfaces. Though the architecture is similar to that of the classical CEO problem, the problem is different from it. In this paper, we address the question of how rate should be allocated among the interfaces in a SIMO-FAWNA, i.e., how fiber capacity should be divided between the interfaces. The interface has noise from two sources, receiver front end and quantizer. We show that an optimal rate allocation is one which ensures that each interface gets enough rate so that its noise is dominated by front end noise rather than by quantizer distortion. This implies higher rates for interfaces seeing higher channel gains. After this rate requirement is met, SIMO-FAWNA capacity is almost invariant to allocation of left over fiber capacity. Hence, large capacity of the optical fiber ensures robustness of SIMO-FAWNA capacity to interface rate allocation. We also show that rather than dynamically changing rate allocation based on channel state, a fixed rate allocation scheme can be adopted with very small loss in capacity. This translates into considerable reduction in FAWNA complexity.
\end{abstract}

\section{INTRODUCTION}

There is a considerable demand for increasingly high-speed communication networks with mobile connectivity. Traditionally, high-speed communication has been efficiently provided through wireline infrastructure, particularly based on optical fiber, where bandwidth is plentiful and inexpensive. However, such infrastructure does not support mobility. Instead, mobile communication is provided by wireless infrastructure, most typically over the radio spectrum. However, limited available spectrum and interference effects limit mobile communication to lower data rates.

In [10], [11], we introduce the concept of a fiber aided wireless network architecture (FAWNA), which allows highspeed mobile connectivity by leveraging the speed of optical networks. Optical networks have speeds typically in hundreds of Megabits/sec or several Gigabits/sec (Gigabit Ethernet, OC48 , OC-192, etc.). In the proposed architecture, the network coverage area is divided into zones such that an optical fiber "bus" passes through each zone. Connected to the end of the fiber is a bus controller/processor, which coordinates use of the fiber as well as connectivity to the outside world. Along the fiber are radio-optical converters (wireless-optical interfaces), which are access points consisting of simple antennas directly

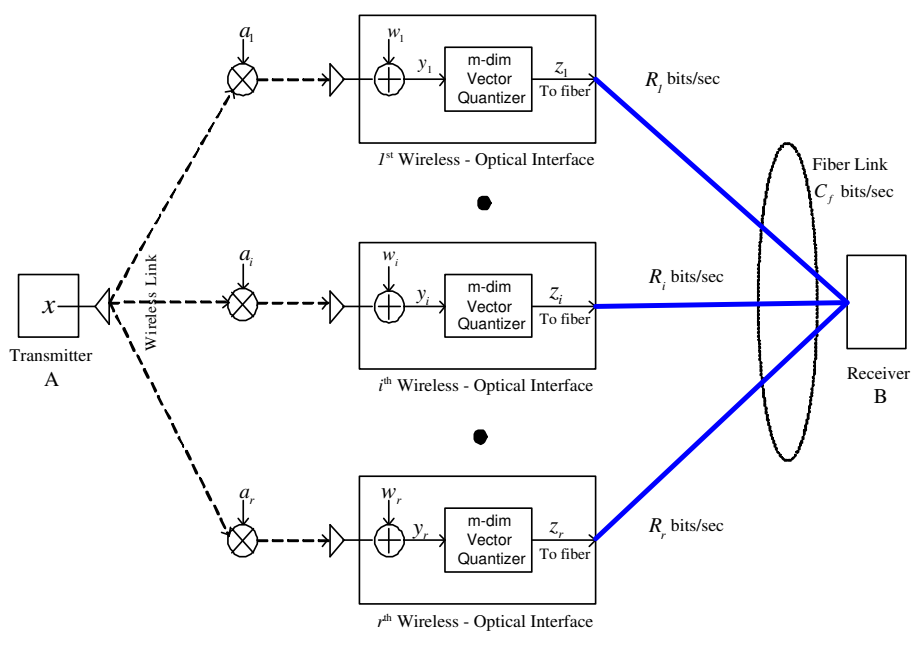

Fig. 1. A SIMO fiber aided wireless network architecture.

connected to the fiber. Each of these antennas harvest the energy from the wireless domain to acquire the full radio bandwidth in their local environment and place the associated waveform onto a subchannel of the fiber. Within the fiber, the harvested signals can be manipulated by the bus controller/processor and made available to all other antennas. In each zone, there may be one or more active wireless nodes. Wireless nodes communicate between one another, or to the outside world, by communicating to a nearby antenna. Thus any node in the network is at most two hops away from any other node, regardless of the size of the network. In general, each zone is generally covered by several antennas, and there may also be wired nodes connected directly to the fiber.

This architecture has the potential to reduce dramatically the interference effects that limit scalability and the energyconsumption characteristics that limit battery life, in pure wireless infrastructure. A FAWNA uses the wireline infrastructure to provide a distributed means of aggressively harvesting energy from the wireless medium in areas where there is a rich, highly vascularized wireline infrastructure and distributing in an effective manner energy to the wireless domain by making use of the proximity of transmitters to reduce interference.

Reference [11] considers a single-input, multiple-output (SIMO) fiber aided wireless network architecture (SIMOFAWNA). Figure 1 shows such a link between two points A 
and $\mathrm{B}$. The various quantities in the figure will be described in detail in the next section. In the two hop link, the first hop is over a wireless channel and the second, over a fiber optic channel. The links we consider are ones where the fiber optic channel capacity is larger than the wireless channel capacity. The transmitter at A transmits information to intermediate wireless-optical interfaces over a wireless SIMO channel. The wireless-optical interfaces then relay this information to the destination, B, over a fiber optic channel. The end-toend design is done to maximize the transmission rate from A to B. Since a FAWNA has a large number of wirelessoptical interfaces, an important design objective is to keep the wireless-optical interface as simple as possible without sacrificing too much in performance.

The problem has a similar setup, but a different objective than the CEO problem [9]. In the CEO problem, the ratedistortion tradeoff is analyzed for a given source that needs to be conveyed to the CEO through an asymptotically large number of agents. Rate-distortion theory is used to analyze the problem. We instead compute the maximum end-to-end rate at which reliable communication is possible. In general, duality between the two problems doesn't exist. Unlike the CEO problem, the number of wireless-optical interfaces is finite and the rate (from interface to receiver B) per interface is high due to the fiber capacity being large. Finite-dimensional, high resolution quantizers are used at the interfaces.

Let us denote the capacities of the wireless and optical channels as $C_{w}(P, W, r)$ and $C_{f}$ bits/sec, respectively, where, $P$ is the average transmit power at $\mathrm{A}, W$ is the wireless transmission bandwidth and $r$ is the number of wirelessoptical interfaces. Since, as stated earlier, we consider links where $C_{w}(P, W, r) \leq C_{f}$, the capacity of a SIMO-FAWNA $C_{\mathrm{SIMO}}\left(P, W, r, C_{f}\right)$ can be upper bounded as

$$
C_{\text {SIMO }}\left(P, W, r, C_{f}\right)<C_{w}(P, W, r) \text { bits/sec. }
$$

One way of communicating over a SIMO-FAWNA is to decode and re-encode at the wireless-optical interfaces. A major drawback of the decode/re-encode scheme is significant loss in optimality because "soft" information in the wireless signal is completely lost by decoding at the wireless-optical interface. Hence, multiple antenna gain is not possible. Moreover, decoding results in the wireless-optical interface having high complexity and the interface requires knowledge of the transmitter code book. In the scheme proposed in [11], the wireless signal at each wireless-optical interface is sampled and quantized before being sent over the fiber. The capacity for this simple forwarding scheme approaches the upper bound (1), exponentially with fiber capacity.

In this paper, we address the problem of interface rate allocation for a SIMO-FAWNA. Reference [11] allocates rates equally among the wireless-optical interfaces irrespective of the fact that wireless channel gains seen by the interfaces are different. This is sub-optimal since FAWNA capacity increases if more rate is allocated to an interface that sees a strong transmit signal than one which sees a weak transmit signal. Hence, interfaces close to the transmitter should have a higher rate than ones further away from it. In this paper, we find the optimal interface rate allocation. We also investigate robustness of FAWNA capacity with respect to interface rate allocation. Unlike the static wireless channel model in [11], we consider a block fading wireless channel model. We analyze the loss from keeping rate allocation fixed (based on wireless channel statistics) rather than dynamically adjusting it according to channel state.

This paper is organized as follows: In section II we describe our model and communication scheme. We analyze interface rate allocation in section III and conclude in section IV. Unless specified otherwise, all logarithms in this paper are to the base 2.

\section{Model AND COMMUNiCATION SCHEME}

There are $r$ wireless-optical interfaces and each of them is equipped with a single antenna. The interfaces relay the wireless signals they receive from the transmitter, to receiver $\mathrm{B}$, over an optical fiber. Communication over the fiber is interference free, which may be achieved, for example, using Time Division Multiple Access (TDMA) or Frequency Division Multiple Access (FDMA).

\section{A. Wireless Channel}

We use a linear model for the wireless channel between A and the wireless-optical interfaces:

$$
\overrightarrow{\mathbf{y}}=\overrightarrow{\mathbf{a}} \mathbf{x}+\overrightarrow{\mathbf{w}},
$$

where, $\mathbf{x} \in \mathcal{C}, \overrightarrow{\mathbf{w}}, \overrightarrow{\mathbf{y}} \in \mathcal{C}^{r}$ are the channel input, additive noise and output, respectively. We assume ergodic block fading where $\overrightarrow{\mathbf{a}} \in \mathcal{C}^{r}$ is the channel state that is random but fixed for a block interval. The channel state changes independently from block to block and is perfectly known at the receiver, B, but not at the transmitter and interfaces. $\mathbf{a}_{i}$ denotes the channel gain for the $i^{t h}$ interface. The additive noise, $\overrightarrow{\mathbf{w}} \sim \mathcal{C N}\left(0, N_{0} I_{r}\right)$, is independent of the channel input and channel state. $N_{0} / 2$ is the double-sided white noise spectral density. The channel input, $\mathbf{x}$, satisfies the average power constraint

$$
E\left[|\mathbf{x}|^{2}\right]=P / W,
$$

where, $P$ and $W$ are the average transmit power at $\mathrm{A}$ and wireless bandwidth, respectively. Hence, the ergodic wireless channel capacity is

$$
C_{w}(P, W, r)=W E\left[\log \left(1+\frac{\|\overrightarrow{\mathbf{a}}\|^{2} P}{N_{0} W}\right)\right],
$$

and $W$ symbols/sec are transmitted over the wireless channel. Thus, using (1), we obtain an upper bound to the SIMOFAWNA ergodic capacity:

$$
C_{\operatorname{SIMO}}\left(P, W, r, C_{f}\right)<W E\left[\log \left(1+\frac{\|\overrightarrow{\mathbf{a}}\|^{2} P}{N_{0} W}\right)\right] .
$$




\section{B. Fiber Optic Channel}

The fiber optic channel between the wireless-optical interfaces and the receiver, $\mathrm{B}$, can reliably support a rate of $C_{f}$ $\mathrm{bits} / \mathrm{sec}$. Communication over the fiber is interference free and the $i^{t h}$ interface communicates at a rate of $R_{i} \mathrm{bits} / \mathrm{sec}$ with receiver B. Now,

$$
\begin{aligned}
& 0<R_{i} \leq C_{f} \quad \text { for } i \in\{1, \ldots, r\}, \\
& \sum_{i=1}^{r} R_{i}=C_{f} .
\end{aligned}
$$

Let us define the set of all rate vectors satisfying these two constraints $(3,4)$ as $\mathcal{S}$. Fiber channel coding is performed at the wireless-optical interface to reliably achieve the rate vectors in $\mathcal{S}$. Note that the code required for the fiber is a very low complexity one. An example of a code that may be used is the $8 \mathrm{~B} 10 \mathrm{~B}$ code, which is commonly used in Ethernet. Hence, fiber channel coding does not significant increase the complexity at the wireless-optical interface. In this work, we assume error free communication over the fiber for all sum rates below fiber capacity. To keep the interfaces simple, source coding is not done at the interfaces. Reference [11] shows that since fiber capacity is large compared to the wireless capacity, the loss from not performing source coding is negligible.

\section{Communication Scheme}

The communication scheme we describe here is similar to the one in [11]. However, we do not limit ourselves to equal interface rate assignment and allow optimization over the set $\mathcal{S}$ of feasible interface rate vectors.

The input to the wireless channel, $\mathbf{x}$, is a zero mean circularly symmetric complex Gaussian random variable, $\mathbf{x} \sim$ $\mathcal{C N}(0, P / W)$. Note that it is this input distribution that achieves the capacity of our wireless channel model. At each wireless-optical interface, the output from the antenna is first converted from passband to baseband and then sampled at the Nyquist rate of $W$ complex samples/sec. The random variable, $\mathbf{y}_{i}$, represents the output from the sampler at the $i^{t h}$ interface. Fixed-rate, memoryless, $m$-dimensional vector quantization is performed on these samples at a rate of $R_{i} / W$ bits/complex sample. The quantized complex samples are subsequently sent over the fiber after fiber channel coding and modulation. Thus, the fiber is required to reliably support a rate of $R_{i}$ bits/sec from the $i^{\text {th }}$ wireless-optical interface to the receiver, B.

The quantizer noise at the $i^{t h}$ interface, $\mathbf{q}_{i}$, is modelled as being additive. Hence, the two-hop channel between A and B can be modelled as:

$$
\overrightarrow{\mathbf{z}}=\overrightarrow{\mathbf{a}} \mathbf{x}+\overrightarrow{\mathbf{w}}+\overrightarrow{\mathbf{q}},
$$

where, $\overrightarrow{\mathbf{q}}=\left[\mathbf{q}_{1}, \ldots, \mathbf{q}_{r}\right]^{T}$, and ${ }^{T}$ denotes transpose. Hence, an interface has noise from two sources, receiver front end and distortion introduced by its quantizer. The quantizer at the interface is an optimal fixed rate memoryless $m$-dimensional high resolution vector quantizer. Hence, its distortion-rate function is given by the Zador-Gersho function [1], [3], [5]:

$$
\begin{aligned}
& E\left[\left|\mathbf{q}_{i}\right|^{2}\right] \\
& \quad=E\left[\left|\mathbf{y}_{i}\right|^{2}\right] M_{m} \beta_{m} 2^{-\frac{R_{i}}{W}} \\
& \quad=\left(N_{0}+\frac{E\left[\left|\mathbf{a}_{i}\right|^{2}\right] P}{W}\right) M_{m} \beta_{m} 2^{-\frac{R_{i}}{W}} .
\end{aligned}
$$

$M_{m}$ is the Gersho's constant which is independent of the distribution of $\mathbf{y}_{i}$ and $\beta_{m}$ is the Zador's factor that depends on the distribution of $\mathbf{y}_{i}$. Since the fiber channel capacity is large, the assumption that the quantizer is a high resolution one, is valid. Hence, for all $i, R_{i} / W \gg 1$. Also, as this quantizer is an optimal fixed rate memoryless vector quantizer, references [2], [3], [4], [6], [7] show that the following hold: $E\left[\mathbf{q}_{i}\right]=0, E\left[\mathbf{z}_{i} \mathbf{q}_{i}^{*}\right]=0$ and $E\left[\mathbf{y}_{i} \mathbf{q}_{i}^{*}\right]=-E\left[\left|\mathbf{q}_{i}\right|^{2}\right]$. Therefore, $E\left[\left|\mathbf{z}_{i}\right|^{2}\right]=E\left[\left|\mathbf{y}_{i}\right|^{2}\right]-E\left[\left|\mathbf{q}_{i}\right|^{2}\right]$. The wireless-optical interfaces have low complexity and do not require knowledge of the transmitter code book. They are also extendable to FAWNAs with large number of transmitters and interfaces and offer adaptability to variable rates, changing channel conditions and node positions. Reference [11] shows that this scheme approaches the capacity upper bound (2) of the architecture, exponentially with fiber capacity. Also, for a given fiber capacity, there is an optimal operating wireless bandwidth and an optimal number of wireless-optical interfaces.

\section{Interface Rate Allocation}

The scheme in [11] allocates equal rates $\left(C_{f} / r\right.$ bits/sec) to the interfaces. This is not optimal since over all quantizer distortion decreases if more rate is allocated to an interface with high signal power than an interface with low signal power. This leads us to two questions: First, how should rates be allocated to the interfaces and second, since channel state varies independently from block to block, is there significant loss in not computing the optimal rate allocation every block? To answer the first question, consider the channel within a block interval. The channel state in this block takes the realization $\overrightarrow{\mathbf{a}}=\vec{a}$. For any rate allocation, $\vec{R}$, the capacity $C_{\mathrm{Q}}(P, W, \vec{a}, \vec{R})$ for this block is given by the following theorem (proof omitted for brevity):

\section{Theorem 1:}

$$
C_{\mathrm{Q}}(P, W, \vec{a}, \vec{R})=W \log \left(\frac{1}{1-\frac{P}{N_{0} W} \vec{v}^{\dagger} M^{-1} \vec{v}}\right)
$$

where, $\vec{v}$ is specified for $i \in\{1, \ldots, r\}$ as

$$
v_{i}=a_{i}\left(1-M_{m} \beta_{m} 2^{-\frac{R_{i}}{W}}\right),
$$

and, $M$ is specified for $i \in\{1, \ldots, r\}, j \in\{1, \ldots, r\}$ as

$$
\begin{aligned}
M_{i j} & =\frac{a_{i} a_{j}^{*} P}{N_{0} W}\left(1-M_{m} \beta_{m} 2^{-\frac{R_{i}}{W}}\right)\left(1-M_{m} \beta_{m} 2^{-\frac{R_{j}}{W}}\right) \\
& \text { for } i \neq j, \\
& \text { for } i=j .
\end{aligned}
$$




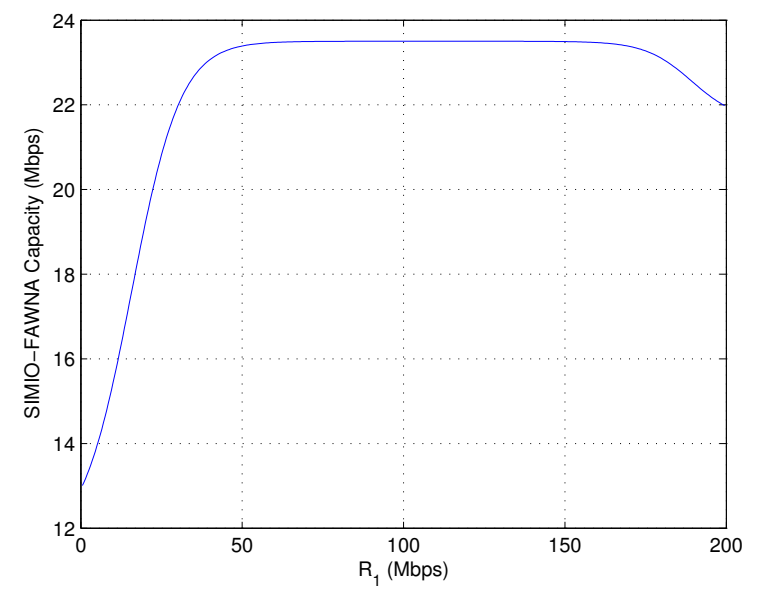

Fig. 2. Interface rate allocation for a two interface SIMO-FAWNA.

From [11], we know that $C_{\mathrm{Q}}(P, W, \vec{a}, \vec{R})$ approaches the capacity upper bound (2) exponentially with sum rate. The optimal rate allocation for this block is given by

$$
\vec{R}^{*}(\vec{a})=\arg \max _{\vec{R} \in \mathcal{S}}\left[C_{\mathbf{Q}}(P, W, \vec{a}, \vec{R})\right] .
$$

To understand optimal rate allocation, let us consider a SIMO-FAWNA with two interfaces ${ }^{1}$, fiber capacity $200 \mathrm{Mbps}$, channel state $\vec{a}=\left[1 \frac{1}{2}\right]^{T}, \frac{P}{N_{0}}=100 \times 10^{6}, W=5 \mathrm{MHz}$ and $M_{m} \beta_{m}=1$. Since $R_{2}=C_{f}-R_{1}$, it suffices to consider the capacity with respect to $R_{1}$ alone. The plot of $C_{\mathrm{Q}}(P, W, \vec{a}, \vec{R})$ with respect to $R_{1}$ is shown in figure 2 .

We can divide the plot into three regions. The first region is from 0 Mbps to 50 Mbps where the first interface has low rate ${ }^{2}$ and the second has high rate. Thus, noise at the first interface is quantizer distortion dominated whereas at the second interface is front end noise dominated. Hence, as we increase the rate for the first interface, the distortion at the first interface decreases and overall capacity increases. The reduction in rate at the second interface due to increase in $R_{1}$ has negligible effect on capacity since front end noise still dominates at the second interface.

The second region is from $50 \mathrm{Mbps}$ to $170 \mathrm{Mbps}$. In this region, the rates for both interfaces are high enough for front end noise to dominate. Since quantizer distortion is low with respect to the front end noise at both interfaces, capacity is almost invariant to rate allocation. Observe that the capacity in this region is higher than that in the first and third regions and, the size of this region is much larger than that of the first and third.

The third region is from $170 \mathrm{Mbps}$ to $200 \mathrm{Mbps}$ and here the first interface has high rate and the second has low rate. Therefore, noise at the first interface is front end noise dominated whereas at the second interface is quantizer distortion

\footnotetext{
${ }^{1}$ Even though we consider a two interface SIMO-FAWNA, results generalize to SIMO-FAWNAs with any number of interfaces.

${ }^{2}$ Whenever we mention "low rate", the rate considered is always high enough for the high resolution quantizer model to be valid.
}

dominated. An increase in rate for the first interface results in decrease in rate for the second interface. This decrease in rate results in increase in quantizer distortion at the second interface, resulting in overall capacity decrease.

The channel gain at the first interface is higher than that at the second interface. Hence, compared to the second interface, the first interface requires more rate to bring its quantizer's distortion below the front end noise. Also, reduction in quantizer distortion at the first interface results in higher capacity gains than reduction in quantizer distortion at the second interface. This can been seen from the asymmetric nature of the plot in figure 2 around $R_{1}=100 \mathrm{Mbps}$.

We see that the optimum interface rate allocation for a FAWNA is to ensure that each interface gets rate enough for it to lower its quantizer distortion to the point where its noise is front end noise dominated. Wireless-optical interfaces seeing higher channel gains require higher rates to bring down their quantizer distortion. After this requirement is met, FAWNA capacity is almost invariant to allocation of left over fiber capacity. This can be seen from the near flat capacity curve from $50 \mathrm{Mbps}$ to $170 \mathrm{Mbps}$ in figure 2. Thus, any interface rate allocation that ensures that noise at none of the wirelessoptical interfaces is quantizer distortion dominated, is optimal. Since fiber capacity is large compared to the wireless capacity, the fraction of fiber capacity needed to bring down the distortion for the interfaces so that none of them is quantizer distortion limited, is small. Therefore, the set of nearoptimal interface rate vectors is large, and there is considerable flexibility in allocating rates across the interfaces. Therefore, we see that large fiber capacity brings robustness to interface rate allocation in a FAWNA. For example, from figure 2, we see that even an equal rate allocation for the two interface SIMO-FAWNA is near-optimal.

We now address the second question posed at the beginning of this section: Since channel state changes independently from block to block, is there significant loss in not computing the optimal rate allocation every block? First, consider the case where interface rate allocation is dynamic, i.e., done in every block. The optimal rate allocation vector is given by (7) and depends on the channel realization. The ergodic capacity of a SIMO-FAWNA with dynamic rate allocation is:

$$
C_{\mathrm{Q}}^{\mathrm{D}}\left(P, W, r, C_{f}\right)=E\left[C_{\mathrm{Q}}\left(P, W, \overrightarrow{\mathbf{a}}, \vec{R}^{*}(\overrightarrow{\mathbf{a}})\right)\right] .
$$

Consider the same two interface SIMO-FAWNA as in the previous question but with channel state $\overrightarrow{\mathbf{a}}=\left[\begin{array}{ll}\mathbf{h}_{1} & \frac{1}{2} \mathbf{h}_{2}\end{array}\right]^{T}$ where, $\mathbf{h}_{1}$ and $\mathbf{h}_{2}$ are i.i.d $\mathcal{C N}(0,1)$. For this wireless-optical channel, we compute $C_{\mathrm{Q}}^{\mathrm{D}}\left(P, W, r, C_{f}\right) \sim 21.4 \mathrm{Mbps}$. Figure 3 shows how the optimal rate for the first interface $R_{1}^{*}$ changes with channel realization (state). Since the average channel gain at the first interface is larger than that at the second, the mean of the observations in the figure is above half the fiber capacity. Dynamic rate allocation involves computation of the optimal rate allocation vector at receiver B and updating the interfaces with optimal values of rates, every coherence block. This considerably increases the complexity in a FAWNA. In order 


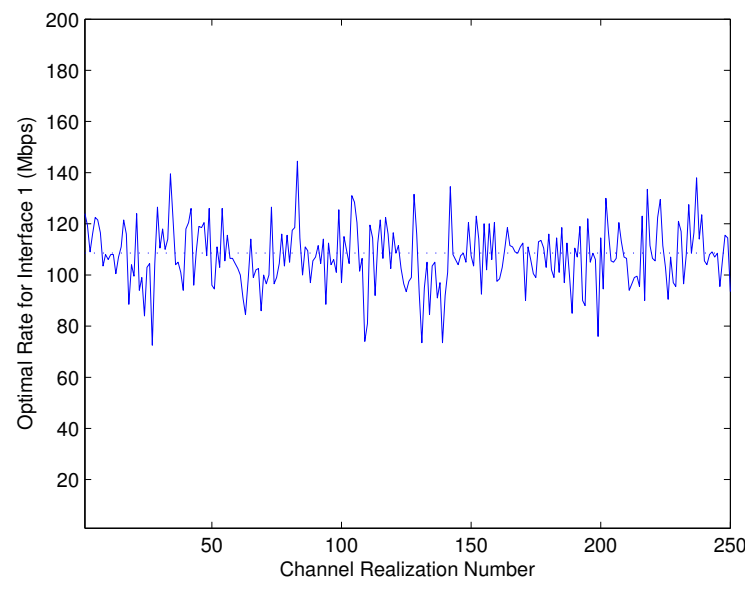

Fig. 3. Dynamic rate allocation.

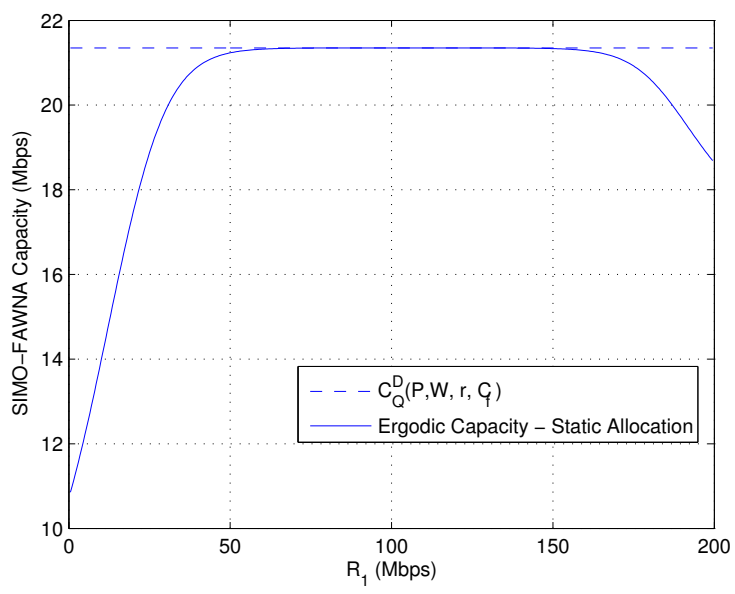

Fig. 4. Near-optimality of static rate allocation.

to simplify, we consider static rate allocation, i.e, interface rate allocation is computed based on wireless channel statistics and fixed forever. The rate allocation vector is chosen as:

$$
\vec{R}_{\mathrm{S}}^{*}=\arg \max _{\vec{R} \in \mathcal{S}} E\left[C_{\mathrm{Q}}(P, W, \overrightarrow{\mathbf{a}}, \vec{R})\right] .
$$

The ergodic capacity of a SIMO-FAWNA with static rate allocation is

$$
C_{\mathrm{Q}}^{\mathrm{S}}\left(P, W, r, C_{f}\right)=E\left[C_{\mathbf{Q}}\left(P, W, \overrightarrow{\mathbf{a}}, \vec{R}_{\mathrm{S}}^{*}\right)\right] .
$$

Note that this is sub-optimal to dynamic rate allocation. For the two interface SIMO-FAWNA, figure 4 shows how ergodic capacity changes with $R_{1}$. Since the ergodic capacity is the capacity averaged over channel realizations, this plot is similar to that in figure 2. From figure 4, we observe that $C_{\mathrm{Q}}^{\mathrm{S}}\left(P, W, r, C_{f}\right)=21.35 \mathrm{Mbps}$ and all rates from $72 \mathrm{Mbps}$ to $142 \mathrm{Mbps}$ are near-optimal for interface 1 .

Note that the loss from static rate allocation is very small. Moreover, the set of near-optimal static rate allocation vectors is large. For this example the loss from not performing dynamic rate allocation is only $50 \mathrm{Kbps}$ or $0.23 \%$ of capacity. Though the SIMO-FAWNA capacity is sensitive to quantizer distortion, large fiber capacity ensures that the interfaces always have enough rate so that they are never distortion limited over the typical set of channel realizations. This robustness of FAWNA capacity to interface rate allocation makes static rate allocation near-optimal. Observe from figure 4 that even equal rate allocation is near-optimal. This near-optimality of static rate allocation translates to considerable reduction in FAWNA complexity.

\section{CONCLUSION}

In this paper, we show that an optimal rate allocation for a SIMO-FAWNA is one which ensures that each interface gets enough rate so that its noise is dominated by front end noise rather than quantizer distortion. Capacity is almost invariant to the way in which left over fiber capacity is allocated. Hence, large fiber capacity ensures robustness of SIMO-FAWNA capacity to interface rate allocation. This robustness has an important implication on design, rather than dynamically change interface rate allocation based on channel state, a fixed rate allocation scheme can be adopted with very small loss in capacity. This results in considerable reduction in FAWNA complexity.

\section{ACKNOWLEDGEMENTS}

The research in this paper is supported by grants NSF CNS-0434974, NSF ANI-0335256 and Stanford University PY-1362.

\section{REFERENCES}

[1] P. L. Zador, "Development and evaluation of procedures for quantizing multivariate distributions", Ph.D. Dissertation, Stanford University, 1963.

[2] J. G. Dunn, "The performance of a class of $n$ dimensional quantizers for a Gaussian source", Proc. Columbia Symp. Signal Transmission Processing, Columbia University, NY 1965.

[3] A. Gersho, "Asymptotically optimal block quantization", IEEE Transactions on Information Theory, vol. IT-25, pp. 373-380, Jul 1979.

[4] J. A. Bucklew and N. C. Gallagher, Jr., "A note on optimum quantization", IEEE Transactions on Information Theory, vol. IT-25, pp. 365-366, May 1979.

[5] P. L. Zador, "Asymptotic quantization error of continuous signals and the quantization dimension", IEEE Transactions on Information Theory, vol. IT-28, pp. 139-148, Mar 1982.

[6] N. C. Gallagher and J. A. Bucklew, "Properties of minimum mean squared error block quantizers", IEEE Transactions on Information Theory, vol. IT-28, pp. 105-107, Jan 1982.

[7] A. Gersho and R. M. Gray, "Vector Quantization and Signal Compression”, Kluwer, Boston, MA, 1992.

[8] D. Hui and D. L. Neuhoff, "On the complexity of scalar quantization", ISIT 1995, p. 372.

[9] T. Berger, Z. Zhang and H. Viswanathan, "The CEO Problem", IEEE Transactions on Information Theory, vol. 42, pp. 887-902, May 1996.

[10] S. Ray, M. Médard and L. Zheng, "Fiber Aided Wireless Network Architecture: A SISO wireless-optical channel", $43^{\text {rd }}$ Allerton Conference on Comunication, Control and Computing, Allerton, Illinois, Sep 2005.

[11] S. Ray, M. Médard and L. Zheng, "A SIMO Fiber Aided Wireless Network Architecture", ISIT 2006, Seattle, WA, Jul 2006. 